\title{
Relationship between retinal morphological findings and visual function in age-related macular degeneration.
}

\section{AUTHOR(S):}

Akagi-Kurashige, Yumiko; Tsujikawa, Akitaka; Oishi, Akio; Ooto, Sotaro; Yamashiro, Kenji; Tamura, Hiroshi; Nakata, Isao; Ueda-Arakawa, Naoko; Yoshimura, Nagahisa

\section{CITATION:}

Akagi-Kurashige, Yumiko ... [et al]. Relationship between retinal morphological findings and visual function in age-related macular degeneration.. Graefe's archive for clinical and experimental ophthalmology 2012, 250(8): 1129-1136

\section{ISSUE DATE:}

2012-08

\section{URL:}

http://hdl.handle.net/2433/160377

\section{RIGHT:}

The final publication is available at www.springerlink.com; この論文は 出版社版でありません。引用の際には出版社版をご確認ご利用くださ $\iota_{\circ}$; This is not the published version. Please cite only the published version. 


\section{Relationship between Retinal Morphological Findings and Visual Function in Age-Related Macular Degeneration}

YUMIKO AKAGI-KURASHIGE, MD; AKITAKA TSUJIKAWA, MD; AKIO OISHI, MD;

SOTARO OOTO, MD; KENJI YAMASHIRO, MD; HIROSHI TAMURA, MD; ISAO

NAKATA, MD; NAOKO UEDA-ARAKAWA, MD; AND NAGAHISA YOSHIMURA, MD

Department of Ophthalmology and Visual Sciences, Kyoto University Graduate School of

Medicine, Kyoto, Japan

Corresponding author: Akitaka Tsujikawa, MD, Department of Ophthalmology, Kyoto

University Graduate School of Medicine, Sakyo-ku, Kyoto 606-8507, Japan

Tel: +81-75-751-3260; Fax: +81-75-752-0933; E-mail: tujikawa@kuhp.kyoto-u.ac.jp 


\section{Abstract}

Background: We aimed to study the retinal morphological findings associated with exudative age-related macular degeneration (AMD) and their association with visual prognosis.

5 Methods: We retrospectively reviewed the medical records of 96 consecutive patients (96 eyes) with exudative AMD. Retinal structural changes were examined using optical coherence tomography (OCT).

Results: Initial OCT examination showed cystoid macular edema in 18 eyes (18.8\%), fibrin exudate in 56 eyes (58.3\%), and hyperreflective foci within the neurosensory retina

10 in 78 eyes (81.3\%). Upon initial examination, an external limiting membrane (ELM) line was detected under the fovea in 64 eyes (66.7\%). Using Pearson's correlation analyses, final visual acuity (VA) was correlated with initial VA $(r=0.61, p<0.001)$, age $(r=0.34, p$ $<0.001)$, initial total foveal thickness $(r=0.41, p<0.001)$, presence of hyperreflective foci $(r=0.40, p<0.001)$, and detection of a foveal ELM line $(r=0.55, p<0.001)$. After

15 multiple regression analysis, final VA correlated with initial VA $(r=0.48, p<0.001)$, initial presence of hyperreflective foci $(r=0.23, p=0.054)$, and detection of a foveal ELM line $(r$ $=0.36, p=0.008)$.

Conclusions: In eyes with exudative AMD, final VA was most correlated with initial VA. 
In addition, the initial integrity of the foveal outer retina was partially correlated with the visual prognosis. The initial ELM condition was associated with good final VA, while the initial presence of hyperreflective foci in the foveal neurosensory retina was associated with poor final VA.

Keywords: age-related macular degeneration, external limiting membrane, hyperreflective foci, optical coherence tomography, polypoidal choroidal vasculopathy 


\section{Introduction}

Age-related macular degeneration (AMD) is a leading cause of blindness worldwide in individuals aged more than 50 years [1]. Although treatment for the disease has progressed dramatically after the development of anti-vascular endothelial growth factor

5 (VEGF) therapy, not all patients achieve good vision [2-4]. A combination of photodynamic therapy (PDT) and anti-VEGF therapy is a promising option [5], but it might not markedly alter the prognosis of AMD [6]. Considering both the physical and financial costs of treatment [7], prediction of the visual prognosis at the patients' initial visits would be very useful to both patients and clinicians.

visual prognosis [8]. For this purpose, we have been trying to make the best use of optical coherence tomography (OCT). Current models of commercially available spectral-domain OCT instruments provide fine images (up to $5-\mu \mathrm{m}$ resolution) of the retinal structure $[9,10]$. Using spectral-domain OCT, Hayashi et al. showed a correlation

15 between retinal structure and function in eyes with AMD that had undergone PDT [11]. In short, the status of the junction between the inner and outer segments of the photoreceptors (IS/OS) [12,13], which is considered to reflect photoreceptor integrity, is associated with visual acuity (VA) [14]. Other authors have confirmed the association of 
IS/OS status with VA [15] and with retinal sensitivity as measured using microperimetry

[16]. In addition to IS/OS, the status of the external limiting membrane (ELM) has also

been reported to be a good indicator of visual function in eyes with AMD [17].

Evaluation of alterations in these retinal layer patterns at the time of the initial

5 examination provides more detailed information about the retinal status $[8,18,19]$.

In addition to the retinal layer pattern, the presence of hyperreflective foci

represents another possible predictive factor of VA [18]. Hyperreflective foci are reported to be present in diabetic macular edema [20], retinal vein occlusion [21], and AMD [18], and have been suggested to be subclinical features of lipoprotein this finding have been reported by other authors; the presence of hyperreflective foci is associated with hard exudate deposition [22]. The breakdown of the blood-retinal barrier as well as the accumulation of hard exudates would both be detrimental to eyes with AMD [18]. correlation between the pretreatment structure of eyes with AMD, and the visual prognosis. However, to date, limited information is available about predictive factors of visual prognosis among the features obtained from initial OCT sections in exudative AMD 
[23-25]. In the study reported herein, we longitudinally investigated OCT images of

eyes with AMD throughout the course of anti-VEGF therapy and examined the relationship between pretreatment features seen on OCT images and the visual prognosis.

\section{Material and methods}

For this observational study, we retrospectively reviewed the medical records of 96 consecutive patients (96 eyes) with exudative AMD who were seen by the Macula

10 Service in the Department of Ophthalmology at Kyoto University Hospital between

January 2008 and October 2009. Inclusion criteria included: (1) symptomatic subfoveal AMD, (2) the presence of choroidal neovascularization (CNV) beneath the foveal center, (3) the presence of macula-related exudative or hemorrhagic features, and (4) a minimum follow-up of 12 months after the initial visit. The current study included eyes

15 with typical AMD and those with polypoidal choroidal vasculopathy (PCV). The diagnosis of PCV was based on indocyanine green angiography, which reveals a branching vascular network that terminates in polypoidal swelling. When both eyes met the inclusion criteria, only the eye with the more active lesion was included in the current 
study. Eyes with other macular abnormalities (i.e., pathologic myopia, retinal angiomatous proliferation, idiopathic CNV, presumed ocular histoplasmosis, angioid streaks, or other secondary CNV) and those with senile cataracts that resulted in poor-quality OCT images were excluded from the current study. This study was approved by the Institutional Review Board at the Kyoto University Graduate School of Medicine and adhered to the tenets of the Declaration of Helsinki.

At the initial visit, each patient underwent a comprehensive ophthalmologic examination, including measurement of best-corrected VA (using the Landolt C test), determination of intraocular pressure, indirect ophthalmoscopy, slit-lamp biomicroscopy with a contact lens, spectral-domain OCT (Spectralis HRA+OCT; Heidelberg Engineering, Heidelberg, Germany), and fluorescein and indocyanine green angiography (HRA2; Heidelberg Engineering). At each scheduled follow-up visit, each patient underwent a complete ophthalmologic examination, including VA measurement, slit-lamp biomicroscopy, indirect fundus ophthalmoscopy, and OCT examination. Fluorescein

15 and indocyanine green angiography were performed as deemed necessary.

After the initial visit, each eye was treated based on disease activity and VA. Eyes with exudative AMD were treated either with PDT combined with intravitreal injection of an anti-VEGF agent, or with intravitreal injections of anti-VEGF agents alone. After the 
initial combined therapy, each eye was considered for retreatment with the combined therapy every 3 months if the eye exhibited residual or recurrent polypoidal lesions upon indocyanine green angiography. In eyes treated with intravitreal injections of anti-VEGF agents alone, additional injections were administered on an as-needed basis.

In the current study, we evaluated the OCT images obtained from all eligible patients both quantitatively and qualitatively. In both the initial and final OCT images, we measured total foveal thickness, defined as the distance between the vitreoretinal interface and the retinal pigment epithelium (Figure 1a). Furthermore, we examined the IS/OS line and the ELM to assess outer foveal photoreceptor layer integrity. The status

10 of the IS/OS line and the ELM line under the fovea was defined as either complete or incomplete. OCT images in eyes with exudative AMD often exhibited subretinal fluid and hemorrhages beneath the neurosensory retina. In addition, most OCT images revealed morphological changes in the neurosensory retina. Cystoid spaces and hyperreflective foci were seen in many cases within the neurosensory retina. Fibrin

15 derived from active CNV or polypoidal lesions was observed as amorphous hyperreflective material, not only in the subretinal space but also within the neurosensory retina. Using horizontal and longitudinal OCT sections, we evaluated whether cystoid macular edema, hyperreflective foci, and fibrin exudates were seen within the $1 \mathrm{~mm} \times 1$ 
$\mathrm{mm}$ area around the fovea (Figure $1 \mathrm{~b})$.

Statistical analysis was performed using software designed for this purpose (IBM

SPSS Statistics Desktop, version 19.0.0; IBM Japan, Tokyo, Japan). All values are presented in terms of means and standard deviation. Best-corrected VA was converted

5 to a logarithm of the minimum angle of resolution (logMAR) equivalent for statistical analysis. Values in typical AMD and PCV were compared using the Student's $t$-test. Bivariate relationships were analyzed using the Pearson's correlation coefficient or the Spearman rank correlation coefficient. Stepwise forward multivariate linear regression analyses were also performed to evaluate the contribution of each initially identifiable

10 factor to final VA. A value of $p<0.05$ was considered statistically significant.

\section{Results}

In the current study, 96 eyes from 96 patients (72 men and 24 women) with exudative

15 AMD who were 57-90 years of age (mean, $73.2 \pm 8.5$ years) were examined (Table 1 ).

Of these 96 eyes, 61 had PCV and 35 had typical AMD. The mean initial VA (logMAR) and foveal thickness were $0.58 \pm 0.49$ and $422.5 \pm 255.9 \mu \mathrm{m}$, respectively; there were no significant differences in these parameters between eyes with typical AMD or PCV $(p=$ 
0.889, $p=0.787$ ). Active CNV lesions were treated with intravitreal injections of anti-VEGF agents in 38 eyes, and with PDT combined with anti-VEGF agents in 37 eyes. No treatments were performed in 21 eyes. The mean number of injections was $4.7 \pm$ 3.2 and mean number of PDT treatments was $1.7 \pm 1.0$. The mean duration from the

5 final treatment to the final examination was $13.4 \pm 9.4$ months. The mean follow-up period was $24.6 \pm 5.7$ months.

At the initial visit, all eyes exhibited exudative changes beneath the neurosensory retina derived from active CNV (subretinal fluid and hemorrhage). In addition, most OCT images revealed morphological changes within the neurosensory retina. Cystoid

10 macular edema was observed in $18.8 \%$ of eyes. Cystoid spaces were usually observed within neurosensory retinas that were in direct contact with the underlying type 2 CNV, whereas detached retinas were rarely seen in any of the cystoid spaces. In $58.3 \%$ of eyes, OCT revealed that the fibrin exudate was an amorphous hyperreflective material within the subretinal space. Fibrin, which was often seen just over the underlying active

15 type 2 CNV or polypoidal lesions, often appeared to infiltrate the overlying neurosensory retina, especially in the outer aspect, resulting in a lack of IS/OS or ELM lines. In these eyes, the ELM seemed to work in some instances as a blocking agent against the exudates (Figure 2). In $81.3 \%$ of eyes, intraretinal hyperreflective foci were seen within 
the neurosensory retina. Intraretinal hyperreflective foci were frequently seen throughout the entire outer retina, not only outside but also beyond the ELM (Figures 1 and 2). In our patients, the integrity of the inner and outer segments of the foveal photoreceptor cells appeared to be compromised due to exudative change from the CNV.

$5 \quad$ At the initial examination, foveal ELM was seen in $66.7 \%$ of the eyes examined, whereas foveal IS/OS was seen in only $22.9 \%$ of the eyes examined.

Table 2 shows the correlations of initial VA with other measurements obtained at the initial examination. Both the total foveal thickness and the presence of cystoid macular edema were correlated with initial VA $(r=0.39, p<0.001 ; r=0.39, p<0.001)$. In

10 addition, foveal IS/OS and ELM were also correlated with initial VA $(r=0.35, p<0.001 ; r$ $=0.48, p<0.001)$.

At the final examination, the mean total thickness was significantly reduced to 305.4 $\pm 224.9 \mu \mathrm{m}(p<0.001)$, while cystoid macular edema was still seen in $11.5 \%$ of the eyes. Despite treatment, restoration of the integrity of the outer photoreceptor layer was limited.

15 Complete detection of the IS/OS and ELM lines was achieved in only $29.1 \%$ and $54.2 \%$ of eyes, respectively. Table 3 shows the correlations between final VA and other measurements obtained at the final examination. The total foveal thickness and presence of cystoid macular edema were correlated with poor final VA $(r=0.27, p<$ 
$0.001 ; r=0.33, p<0.001)$, whereas detection of foveal IS/OS and ELM lines was

correlated with good final VA $(r=0.57, p<0.001 ; r=0.58, p<0.001)$. There were no differences in final VA between eyes with typical AMD and PCV $(p=0.149)$.

Table 4 shows the correlations of final VA with measurements obtained at the initial examination (Pearson's correlation analyses); of these, initial VA showed the closest correlation with final VA $(r=0.61, p<0.001)$. Age $(r=0.34, p<0.001)$, total foveal thickness $(r=0.40, p<0.001)$, and the presence of hyperreflective foci or cystoid macular edema $(r=0.26, p=0.012)$ at the initial visit also correlated with final VA. In addition, the initial detection of the foveal IS/OS and ELM lines was correlated with final

$10 \operatorname{VA}(r=0.42, p<0.001$; and $r=0.55, p<0.001$, respectively $)$. However, there were no differences in final VA between treatment types $(p=0.637)$. Table 5 shows the correlations between VA and the measurements obtained at the initial examination in each group, stratified by treatment. In each group, while the correlations had similar tendencies, some were not statistically significant, perhaps due to the small number of eyes.

Table 6 shows the correlations between final VA and the measurements obtained at the initial examination after multiple regression analysis. By multiple regression analysis, final VA was correlated with initial VA $(r=0.48, p<0.001)$ and the detection of a 
foveal ELM line $(r=0.33, p=0.008)$. The initial presence of cystoid macular edema was associated with initial poor VA $(r=0.39, p<0.001)$, but had no significant correlation with final visual function. On the other hand, the initial presence of hyperreflective foci had no significant correlation with initial VA $(r=0.11, p=0.261)$, but showed a marginal correlation with final VA $(r=0.23, p=0.054)$

\section{Discussion}

In the present study, we evaluated the morphological findings of the retina associated with exudative AMD and found that initial ELM status and the presence of hyperreflective foci are associated with the visual prognosis. Although the predictive power was inferior to that of initial VA, these parameters at the time of the initial examination can be of help in predicting the visual prognosis.

Although both ELM and IS/OS status were correlated with visual prognosis in

15 bivariate relationship analysis, the multiple regression model showed that only ELM, and not IS/OS status, contributes to visual prognosis. This finding can be explained, at least in part, by the previously held notion that the IS/OS change is too sensitive for use in the evaluation of diseases that cause severe retinal damage, such as exudative AMD [17]. 
In the current study, of the 96 eyes, IS/OS and ELM lines just beneath the fovea were confirmed in 22 eyes (22.9\%) and 64 eyes (66.7\%), respectively, suggesting that the IS/OS is impaired relatively early in the course of AMD. In fact, the ELM line was always confirmed when an IS/OS line was detectable. This finding is consistent with the

5 findings of previous studies that examined other macular diseases (e.g., macular hole [26], retinal detachment [27]). Taken together, these facts indicate that the IS/OS is more susceptible and is disrupted in earlier stages than is the ELM. Photoreceptor damage appears as disruption of the IS/OS at first and subsequently of the ELM in these conditions, so the evaluation of the ELM and of the IS/OS depends on the severity of the disease.

An intact ELM might indicate the preservation of the anatomic barrier as well as photoreceptor integrity. The ELM consists of the zonula adherens between the Müller cells and the photoreceptors at the base of the outer segments; this junction is not as tight as that of the zonula occludens, but it does limit the movement of large molecules

15 [28]. As shown in Figure 2, some eyes demonstrated termination of subretinal fibrin at the ELM border, suggesting that the ELM actually acts as a barrier to subretinal proteins or lipids. The prevention of molecular invasion and the subsequent retinal fluid accumulation would have beneficial effects on visual prognosis, providing another reason 
to study ELM status and its relationship with visual prognosis.

The present study also showed that hyperreflective foci could possibly predict vision, as the presence of hyperreflective foci was negatively associated with the visual prognosis. Although hyperreflective foci are not a functional retinal component and

5 subfoveal hard exudate accumulation was not a cause of poor visual prognosis, we believe that the presence of hyperreflective foci are a hallmark of blood-retinal barrier function, and thus reflect visual outcome. Hyperreflective foci were visible before treatment in many eyes (81.3\%) but tended to disappear as the exudative changes improved (40.6\% at final examination). An earlier report also suggested that the

10 presence of hyperreflective foci reflects blood-retinal barrier impairment [20]. It is possible that eyes with hyperreflective foci at the initial examination had more active CNV and more severe blood-retinal barrier damage. In other words, the small percentage of cases without hyperreflective foci had milder CNV and milder blood-retinal barrier damage; this difference in the degree of blood-retinal barrier damage might have affected

15 visual prognosis.

There are many limitations to the present study, including the retrospective study design, the relatively small study population from a single institution, and the variety of treatment regimens used. While the heterogeneity in disease types might have affected 
the results, there were no differences in initial and final VA between eyes with typical AMD and PCV. In the current study, eyes with exudative AMD were treated with intravitreal injections of anti-VEGF agents with or without PDT. When stratified by treatment, the correlations showed similar tendencies, some of which were not

5 statistically significant, perhaps due to the small number of eyes. Eyes treated with combination therapy showed the lowest association between the initial condition of the outer retina and final VA. While the phototoxic effect of PDT might be involved in this low correlation, the exact reason is not unclear. It is possible that the treatment regimen used have some effect on the visual prognosis. Another limitation is the very nature of OCT examinations. The device used depicts only a difference in light reflectance. Furthermore, we are not completely sure what the presence of an intact ELM or hyperreflective foci implies. Both of these are issues that need to be addressed in future studies. Although initial VA is most strongly associated with the visual prognosis, the initial condition of the outer retina may be of

15 help in predicting the visual prognosis in eyes with AMD. This information will help clinicians provide appropriate information to their patients. A further prospective study is necessary to establish the factors that predict visual prognosis in eyes with exudative AMD. 


\section{References}

1. Yannuzzi LA, Friedman R, Fine SL, Gass JDM, Gitter KA, Orth DH, Singerman LJ (1988) Symposium on age-related macular degeneration. Bull N Y Acad Med 64: 955-1013 (2006) Ranibizumab for neovascular age-related macular degeneration. N Engl J Med 355: 1419-1431

3. Brown DM, Kaiser PK, Michels M, Soubrane G, Heier JS, Kim RY, Sy JP, Schneider S (2006) Ranibizumab versus verteporfin for neovascular age-related macular degeneration. N Engl J Med 355: 1432-1444

4. Brown DM, Michels M, Kaiser PK, Heier JS, Sy JP, lanchulev T (2009) Ranibizumab versus verteporfin photodynamic therapy for neovascular age-related macular degeneration: two-year results of the ANCHOR study. Ophthalmology 116: 57-65

15 5. Heier JS, Boyer DS, Ciulla TA, Ferrone PJ, Jumper JM, Gentile RC, Kotlovker D, Chung CY, Kim RY (2006) Ranibizumab combined with verteporfin photodynamic therapy in neovascular age-related macular degeneration: year 1 results of the FOCUS Study. Arch Ophthalmol 124: 1532-1542 
6. Schmidt-Erfurth UM, Pruente C (2007) Management of neovascular age-related macular degeneration. Prog Retin Eye Res 26: 437-451

7. Oishi A, Mandai M, Nishida A, Hata M, Matsuki T, Kurimoto Y (2011) Remission and dropout rate of anti-VEGF therapy for age-related macular degeneration. Eur J Ophthalmol, in press

8. Coscas G, Coscas F, Vismara S, Zourdani A, Li Calzi Cl (2009) OCT interpretation. In: Coscas G, Coscas F, Vismara S, Zourdani A, Li Calzi Cl (eds) Optical coherence tomography in age-related macular degeneration. Springer-Verlag, Heidelberg, pp 97-170

109 Ko TH, Fujimoto JG, Schuman JS, Paunescu LA, Kowalevicz AM, Hartl I, Drexler W, Wollstein G, Ishikawa H, Duker JS (2005) Comparison of ultrahigh- and standard-resolution optical coherence tomography for imaging macular pathology. Ophthalmology 112: 1922-1935

10. Drexler W, Sattmann H, Hermann B, Ko TH, Stur M, Unterhuber A, Scholda C, Findl O, Wirtitsch M, Fujimoto JG, Fercher AF (2003) Enhanced visualization of macular pathology with the use of ultrahigh-resolution optical coherence tomography. Arch Ophthalmol 121: 695-706 
11. Hayashi H, Yamashiro K, Tsujikawa A, Ota M, Otani A, Yoshimura N (2009) Association between foveal photoreceptor integrity and visual outcome in neovascular age-related macular degeneration. Am J Ophthalmol 148: 83-89

12. Gloesmann M, Hermann B, Schubert C, Sattmann H, Ahnelt PK, Drexler W (2003) Histologic correlation of pig retina radial stratification with ultrahigh-resolution optical coherence tomography. Invest Ophthalmol Vis Sci 44: 1696-1703

13. Anger EM, Unterhuber A, Hermann B, Sattmann H, Schubert C, Morgan JE, Cowey A, Ahnelt PK, Drexler W (2004) Ultrahigh resolution optical coherence tomography of the monkey fovea. Identification of retinal sublayers by correlation with semithin histology sections. Exp Eye Res 78: 1117-1125

14. Costa RA, Calucci D, Skaf M, Cardillo JA, Castro JC, Melo LA Jr, Martins MC, Kaiser PK (2004) Optical coherence tomography 3: Automatic delineation of the outer neural retinal boundary and its influence on retinal thickness measurements. Invest Ophthalmol Vis Sci 45: 2399-2406

15 15. Sayanagi K, Sharma S, Kaiser PK (2009) Photoreceptor status after antivascular endothelial growth factor therapy in exudative age-related macular degeneration. Br J Ophthalmol 93: 622-626 
16. Landa G, Su E, Garcia PM, Seiple WH, Rosen RB (2011) Inner segment-outer segment junctional layer integrity and corresponding retinal sensitivity in dry and wet forms of age-related macular degeneration. Retina 31: 364-370

17. Oishi A, Hata M, Shimozono M, Mandai M, Nishida A, Kurimoto Y (2010) The significance of external limiting membrane status for visual acuity in age-related macular degeneration. Am J Ophthalmol 150: 27-32

18. Coscas G, Coscas F, Vismara S, Zourdani A, Li Calzi Cl (2009) Clinical features and natural history of AMD. In: Coscas G, Coscas F, Vismara S, Zourdani A, Li Calzi Cl (eds): Optical coherance tomography in age-related macular degeneration. Springer-Verlag, Heidelberg, pp 171-274

19. Coscas F, Coscas G, Souied E, Tick S, Soubrane G (2007) Optical coherence tomography identification of occult choroidal neovascularization in age-related macular degeneration. Am J Ophthalmol 144: 592-599

20. Bolz M, Schmidt-Erfurth U, Deak G, Mylonas G, Kriechbaum K, Scholda C (2009) extravasation in diabetic macular edema. Ophthalmology 116: 914-920 
21. Ogino K, Murakami T, Tsujikawa A, Miyamoto K, Sakamoto A, Ota M, Yoshimura $N$ (2011) Characteristics of optical coherence tomographic hyperreflective foci in retinal vein occlusion. Retina, in press

22. Ota M, Nishijima K, Sakamoto A, Murakami T, Takayama K, Horii T, Yoshimura N (2010) Optical coherence tomographic evaluation of foveal hard exudates in patients with diabetic maculopathy accompanying macular detachment. Ophthalmology 117: 1996-2002

23. Shah AR, Del Priore LV (2009) Natural history of predominantly classic, minimally classic, and occult subgroups in exudative age-related macular degeneration.

24. Kaiser PK, Brown DM, Zhang K, Hudson HL, Holz FG, Shapiro H, Schneider S, Acharya NR (2007) Ranibizumab for predominantly classic neovascular age-related macular degeneration: subgroup analysis of first-year ANCHOR results. Am J Ophthalmol 144: 850-857

15 25. Boyer DS, Antoszyk AN, Awh CC, Bhisitkul RB, Shapiro H, Acharya NR (2007) Subgroup analysis of the MARINA study of ranibizumab in neovascular age-related macular degeneration. Ophthalmology 114: 246-252 
26. Theodossiadis PG, Grigoropoulos VG, Theodossiadis GP (2011) The significance of the external limiting membrane in the recovery of photoreceptor layer after successful macular hole closure: a study by spectral domain optical coherence tomography. Ophthalmologica 225: 176-184

5 27. Wakabayashi T, Oshima Y, Fujimoto H, Murakami Y, Sakaguchi H, Kusaka S, Tano Y (2009) Foveal microstructure and visual acuity after retinal detachment repair: imaging analysis by Fourier-domain optical coherence tomography. Ophthalmology 116: 519-528

28. Marmor MF (1999) Mechanisms of fluid accumulation in retinal edema. Doc 


\section{Figure Legends}

Fig. 1. A. Horizontal image of the fovea obtained using optical coherence tomography (OCT) of an eye with active exudative age-related macular degeneration. Using an initial OCT image, 3 measurements were made in the fovea, including total foveal

5 thickness, continuity of the external limiting membrane (ELM; white arrowheads), and continuity of the junction between the inner and outer photoreceptor segments (IS/OS; yellow arrowheads). B. Cross-sectional image of the foveal region obtained using OCT. Using these OCT sections, we determined whether cystoid macular edema (white arrow), hyperreflective foci (yellow arrows), or fibrin exudate (yellow arrowheads) were seen within the $1 \mathrm{~mm} \times 1 \mathrm{~mm}$ square area around the fovea.

Fig. 2. Changes in retinal structure seen in polypoidal choroidal vasculopathy. Initial fluorescein angiography. B. Initial indocyanine green angiography shows numerous polypoidal lesions. C. Initial fundus photograph exhibiting reddish-orange

15 nodules and an adjacent detachment of the pigment epithelium. D. Fundus photograph at final visit. E. Sectional image obtained using optical coherence tomography (OCT) at the initial visit. Numerous hyperreflective foci (yellow arrows), as well as subretinal fluid/fibrin (yellow asterisk) are seen. The fibrin appears to have infiltrated the outer 
retina but appears to be blocked at the external limiting membrane (white arrowheads).

The structure of the neurosensory retina seems to be relatively well preserved. The line of the external limiting membrane is detectable under the fovea. The visual acuity was

0.3 by the Landolt chart. F. Sectional image obtained using OCT at the final visit. The

$5 \quad$ visual acuity was 0.9 by the Landolt chart.

Fig. 3. Changes in retinal structure seen in typical age-related macular degeneration.

A. Initial fluorescein angiography shows minimally classic choroidal neovascularization

(CNV). B. Initial indocyanine green angiography. C. Fundus photograph at initial visit

10 shows subfoveal exudate with surrounding subretinal hemorrhage. D. Fundus photograph at final visit shows subfoveal fibrous tissue. E. Sectional image obtained using optical coherence tomography (OCT) at the initial visit. Hyperreflective foci (yellow arrows) and cystoid macular edema (white arrow) are seen within the neurosensory retina. Subretinal fluid/fibrin (yellow asterisk) is seen subfoveally. The

15 line of the external limiting membrane is not detectable under the fovea. Visual acuity was 0.2 by the Landolt chart. F. Sectional image obtained using OCT at the final visit. Sectional OCT image at 20 months shows thick subfoveal deposit. The visual acuity was 0.1 by the Landolt chart. 
Table 1. Characteristics of the study population

Number of eyes 96

Age (years; mean [SD $\left.{ }^{a}\right]$ )

$73.2(8.5)$

Gender (women/men)

$24 / 72$

Initial examination

Visual acuity (logMAR ${ }^{\mathrm{b}}$; mean [SD])

$0.58(0.49)$

Total foveal thickness ( $\mu \mathrm{m}$; mean [SD])

$422.5(255.9)$

Detection of IS/OS ${ }^{c}$ under the fovea (complete/incomplete)

$22 / 74$

Detection of ELM ${ }^{d}$ under the fovea (complete/incomplete)

$64 / 32$

Cystoid macular edema, number of eyes (\%)

$18(18.8)$

Hyperreflective foci, number of eyes (\%)

Fibrin, number of eyes (\%)

Follow-up (months; mean [SD])

Treatment

Photodynamic therapy (\%)

Anti-vascular endothelial growth factor therapy (\%)

Final examination

Visual acuity (logMAR; mean [SD])

Total foveal thickness ( $\mu \mathrm{m}$; mean [SD])

$305.4(224.9)$

Detection of IS/OS under the fovea (complete/incomplete)

Detection of ELM under the fovea (complete/incomplete)

$52 / 44$

Cystoid macular edema, number of eyes (\%)

Hyperreflective foci, number of eyes (\%)

Fibrin, number of eyes (\%) IS/OS, junction between the inner and outer photoreceptor segments; ${ }^{\mathrm{d}} \mathrm{ELM}$, external limiting membrane 
Table 2. Association between initial visual acuity and other measurements obtained at initial examination

\begin{tabular}{llc}
\hline & $r$ & $p$ value \\
\hline Age & 0.20 & 0.048 \\
Gender & 0.03 & 0.782 \\
Diabetes mellitus & 0.04 & 0.720 \\
Hypertension & 0.08 & 0.413 \\
Smoking & 0.09 & 0.370 \\
Type of disease & 0.03 & 0.787 \\
Total foveal thickness & 0.39 & $<0.001$ \\
Cystoid macular edema & 0.39 & $<0.001$ \\
Hyperreflective foci & 0.11 & 0.261 \\
Fibrin & 0.05 & 0.603 \\
Detection of IS/OS & & $<0.001$ \\
Detection of ELM ${ }^{b}$ under the fovea & 0.35 & $<0.001$ \\
\hline
\end{tabular}

${ }^{\mathrm{a}} \mathrm{S} / \mathrm{OS}$, junction between the inner and outer photoreceptor segments; ${ }^{\mathrm{b}} \mathrm{ELM}$, external limiting membrane 
Table 3. Association between final visual acuity and other measurements obtained at final examination

\begin{tabular}{|c|c|c|}
\hline & r & $p$ value \\
\hline Age & 0.34 & $<0.001$ \\
\hline Gender & 0.03 & 0.770 \\
\hline Follow-up period & 0.03 & 0.787 \\
\hline Diabetes mellitus & 0.06 & 0.589 \\
\hline Hypertension & 0.02 & 0.827 \\
\hline Smoking & 0.01 & 0.908 \\
\hline Total foveal thickness & 0.27 & $<0.001$ \\
\hline Cystoid macular edema & 0.33 & $<0.001$ \\
\hline Hyperreflective foci & 0.16 & 0.120 \\
\hline Fibrin & 0.12 & 0.258 \\
\hline Detection of IS/OS ${ }^{a}$ under the fovea & 0.57 & $<0.001$ \\
\hline Detection of ELM ${ }^{b}$ under the fovea & 0.58 & $<0.001$ \\
\hline
\end{tabular}

${ }^{\mathrm{a}} \mathrm{S} / \mathrm{OS}$, junction between the inner and outer photoreceptor segments; ${ }^{\mathrm{b}} \mathrm{ELM}$, external limiting membrane

${ }^{*}$ Fisher's Least Significant Difference test 
Table 4. Associations between final visual acuity and measurements obtained at initial examination and during treatment

\begin{tabular}{|c|c|c|}
\hline & $r$ & $p$ value \\
\hline Age & 0.34 & $<0.001$ \\
\hline Gender & 0.03 & 0.770 \\
\hline Diabetes mellitus & 0.06 & 0.589 \\
\hline Hypertension & 0.02 & 0.827 \\
\hline Smoking & 0.01 & 0.908 \\
\hline Type of disease & 0.15 & 0.149 \\
\hline Visual acuity $\left(\log M A R^{a}\right)$ & 0.61 & $<0.001$ \\
\hline Total foveal thickness & 0.40 & $<0.001$ \\
\hline Cystoid macular edema & 0.34 & $<0.001$ \\
\hline Hyperreflective foci & 0.26 & 0.012 \\
\hline Fibrin & 0.06 & 0.513 \\
\hline Detection of IS/OS ${ }^{b}$ under the fovea & 0.42 & $<0.001$ \\
\hline Detection of ELM ${ }^{c}$ under the fovea & 0.55 & $<0.001$ \\
\hline Treatment & - & $0.637^{*}$ \\
\hline
\end{tabular}

${ }^{a} \log M A R$, logarithm of the minimum angle of resolution; ${ }^{b} \mathrm{IS} / \mathrm{OS}$, junction between inner and outer segments of the photoreceptors; ' ELM, external limiting membrane

${ }^{*}$ Fisher's Least Significant Difference test 
Table 5. Association between final visual acuity and other measurements obtained at initial examination in each group, stratified by treatment

\begin{tabular}{|c|c|c|c|c|c|c|}
\hline & \multicolumn{2}{|c|}{$\begin{array}{l}\text { No treatment } \\
\quad(n=21)\end{array}$} & \multicolumn{2}{|c|}{$\begin{array}{c}\text { Photodynamic } \\
\text { therapy with } \\
\text { anti-VEGF agents } \\
\quad(\mathrm{n}=37)\end{array}$} & \multicolumn{2}{|c|}{$\begin{array}{c}\text { Anti-VEGF }^{a} \\
\text { agents } \\
(\mathrm{n}=38)\end{array}$} \\
\hline & $r$ & $p$ value & $r$ & $p$ value & $r$ & $p$ value \\
\hline Age & 0.50 & 0.022 & 0.32 & 0.058 & 0.27 & 0.106 \\
\hline Gender & 0.40 & 0.073 & 0.23 & 0.177 & 0.03 & 0.869 \\
\hline Diabetes mellitus & 0.09 & 0.712 & 0.27 & 0.101 & 0.22 & 0.175 \\
\hline Hypertension & 0.13 & 0.576 & 0.15 & 0.367 & 0.04 & 0.793 \\
\hline Smoking & 0.02 & 0.926 & 0.12 & 0.481 & 0.14 & 0.397 \\
\hline Visual acuity (logMAR $\left.{ }^{b}\right)$ & 0.76 & $<0.001$ & 0.48 & 0.003 & 0.62 & $<0.001$ \\
\hline Total foveal thickness & 0.45 & 0.042 & 0.36 & 0.029 & 0.40 & 0.013 \\
\hline Cystoid macular edema & NA & NA & 0.26 & 0.117 & 0.49 & 0.002 \\
\hline Hyperreflective foci & 0.38 & 0.090 & 0.01 & 0.968 & 0.28 & 0.093 \\
\hline Fibrin & 0.01 & 0.957 & 0.07 & 0.666 & 0.12 & 0.469 \\
\hline $\begin{array}{l}\text { Detection of IS/OS } \\
\text { under the fovea }\end{array}$ & 0.47 & 0.034 & 0.32 & 0.055 & 0.46 & 0.004 \\
\hline $\begin{array}{l}\text { Detection of ELM }{ }^{d} \text { under } \\
\text { the fovea }\end{array}$ & 0.71 & $<0.001$ & 0.26 & 0.117 & 0.65 & $<0.001$ \\
\hline
\end{tabular}

${ }^{a}$ VEGF, vascular endothelial growth factor; ${ }^{b} \operatorname{logMAR}$, logarithm of the minimum angle of resolution; ' IS/OS, junction between inner and outer photoreceptor segments; ${ }^{\mathrm{d}} \mathrm{ELM}$, external limiting membrane

No eyes exhibited cystoid macular edema at the initial visit in the no treatment group. 
Table 6. Association between final visual acuity and measurements obtained at initial examination, evaluated by multiple regression analysis

\begin{tabular}{|c|c|c|}
\hline & Partial regression coefficient & $p$ value \\
\hline Age & 0.01 & 0.036 \\
\hline Gender & $N A^{a}$ & - \\
\hline Diabetes mellitus & NA & - \\
\hline Hypertension & NA & - \\
\hline Smoking & NA & - \\
\hline Type of disease & NA & - \\
\hline Visual acuity $\left(\log M A R^{b}\right)$ & 0.48 & $<0.001$ \\
\hline Total foveal thickness & $<0.01$ & 0.292 \\
\hline Cystoid macular edema & 0.02 & 0.856 \\
\hline Hyperreflective foci & 0.23 & 0.054 \\
\hline Fibrin & NA & - \\
\hline Detection of IS/OS ${ }^{c}$ under the fovea & 0.13 & 0.279 \\
\hline Detection of ELM ${ }^{d}$ under the fovea & 0.33 & 0.008 \\
\hline
\end{tabular}




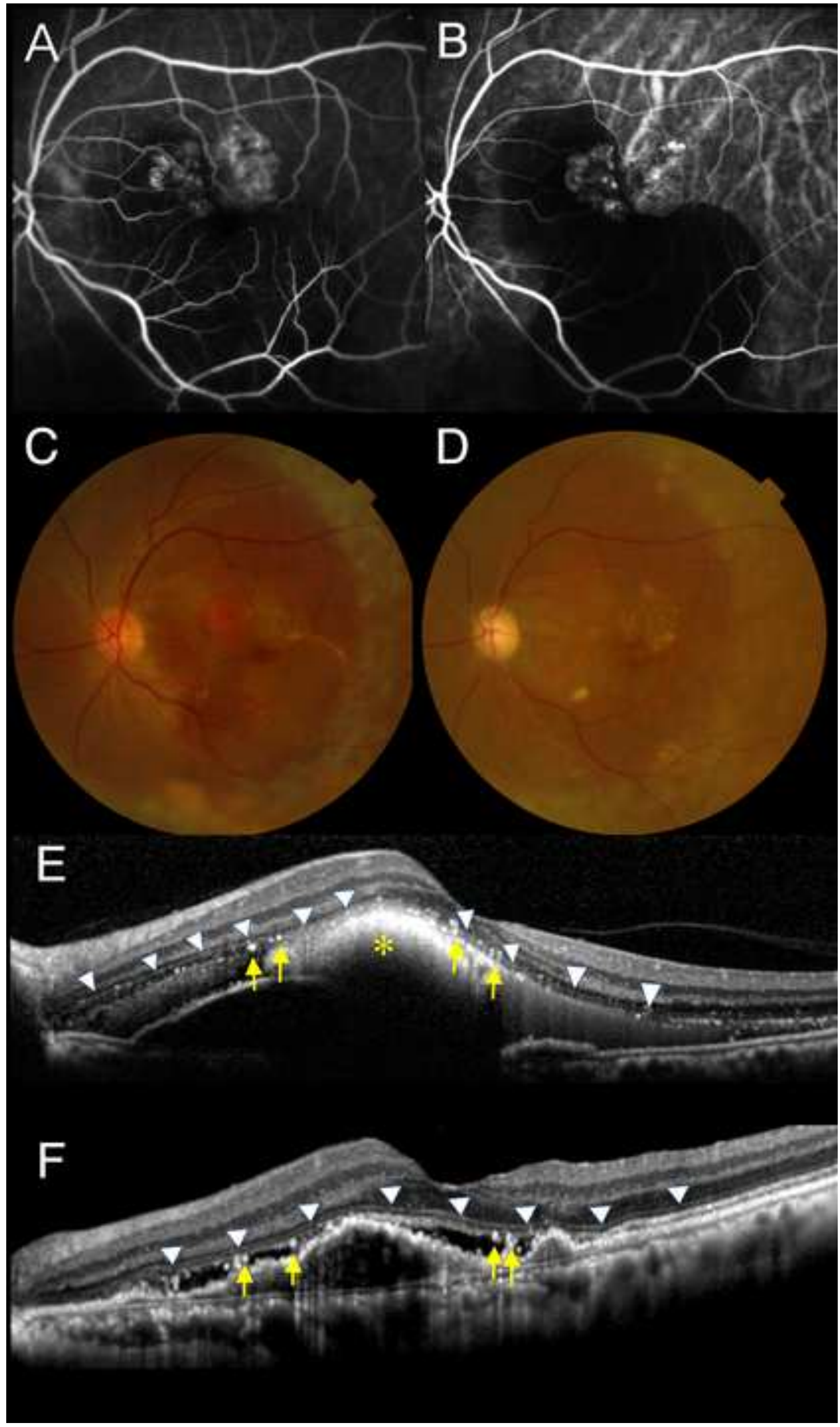



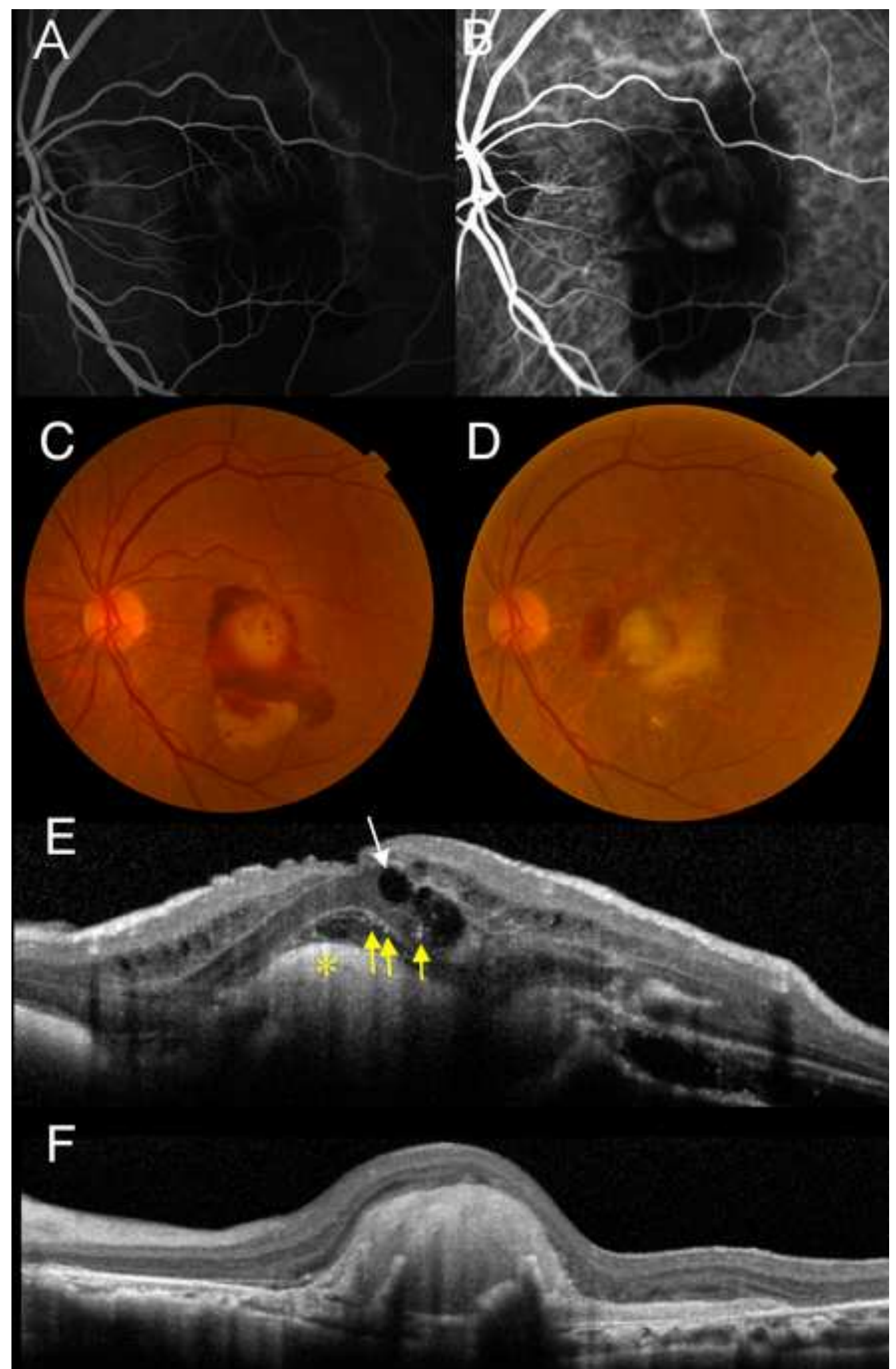